\title{
Terapia grupal de comportamiento mejora la calidad de vida en hombres post prostatectomía radical
}

\author{
FG Perchon L*,**, CR Palma $\mathrm{P}^{* *}$, Magna LA***, Dambros $\mathrm{M}^{* * * *}$.
}

*Division of Geriatric Urology, Federal University of São Paulo, **Division of Urology, State University of Campinas, ***Department of Human Genetics, State University of Campinas, Brasil.

Actas Urol Esp. 2008;32(7):686-690

\section{RESUMEN}

TERAPIA DE COMPORTAMIENTO EN GRUPO PARA MEJORAR LA CALIDAD DE VIDA EN PACIENTES TRAS PROSTATECTOMÍA RADICAL

Antecedentes: Este estudio valoró la eficacia de la terapia de comportamiento en grupo para mejorar la calidad de vida, durante 24 semanas, entre hombres que han sido tratados de cáncer de próstata localizado por medio de prostatectomía radical y presentan disfunciones relacionadas con el tratamiento: incontinencia urinaria y disfunción eréctil.

Pacientes y Métodos: Se asignaron 30 hombres a un grupo de terapia de comportamiento durante 24 semanas. Esta participación se diseñó para mejorar la calidad de vida ayudando a los participantes a identificar y controlar de forma efectiva las experiencias traumáticas Se centró en las secuelas relacionadas con el tratamiento de prostatectomía radical.

Las diferencias en las variables se compararon al principio y al final del estudio por medio de la prueba t de Student para muestras pareadas.

Se llevaron a cabo múltiples análisis por etapas de regresión lineal múltiple siguiendo el análisis de correlación bivariante de Pearson. Esto se logró con todas las predicciones (es decir la percepción de salud general, e impacto en la disfunción eréctil e incontinencia urinaria) y las covariables importantes (es decir la edad, la situación trabajo/jubilación, la adicción al alcohol, la actitud hacia el cáncer y la cirugía, la satisfacción sexual, y los planes futuros.

Resultados: En todos los pacientes el tiempo transcurrido desde la cirugía a la asistencia al grupo de terapia del comportamiento superó los 24 meses. No hubo correlación significativa entre este momento y ninguna de las variables. Las puntuaciones de la percepción general de salud disminuyeron en el final del estudio $(p=0,000)$, al igual que la puntuación de la incontinencia urinaria ( $p=0,023)$, lo que indica mejoría en ambos factores. La diferencia en las puntuaciones de la incontinencia urinaria se correlaciona significativamente y negativamente con la edad $(\mathrm{p}=0,04)$ y el trabajo/jubilación $(\mathrm{p}=0,05)$. Las múltiples etapas de regresión mostraron que la edad es la variable más importante $\left(\mathrm{r}^{2}=26,0 \%\right)$. Teniendo en cuenta la edad y el trabajo/jubilación al mismo tiempo, hubo un aumento del $10,3 \%\left(\mathrm{r}^{2}=36,3 \%\right)$. La disfunción eréctil aumentó en el final del estudio $(\mathrm{p}=0,000)$, y la diferencia entre las puntuaciones de la disfunción eréctil se correlacionaron positiva y significativamente solamente con la satisfacción sexual $(\mathrm{p}=0,029)$, lo que significa que la satisfacción sexual anterior tuvo una influencia positiva sobre la disfunción eréctil $\left(\mathrm{r}^{2}=15,8 \%\right)$.

Conclusión: La terapia de comportamiento en grupo de 24 semanas fue eficaz para mejorar la percepción de calidad de vida, entre los hombres tratados con prostatectomía radical. Hubo cambios asociados con la terapia, especialmente la mejoría en la incontinencia urinaria y la disfunción eréctil.

Palabras clave: Terapia de comportamiento en grupo. Carcinoma de próstata. Calidad de vida.

\section{ABSTRACT}

BEHAVIORAL GROUP-BASED THERAPY IMPROVES GUALITY OF LIFE IN MEN RECOVERING FROM RADICAL PROSTATECTOMY

Background: This study evaluated the efficacy of 24-week, group-based, behavioral therapy in improving quality of life $(\mathrm{QoL})$ among men treated for localized prostate carcinoma (PC) by means of radical prostatectomy (RP) who presented treatment-related dysfunctions: urinary incontinence (UI) and erectile dysfunction (ED).

Patients and methods: Thirty men were assigned to 24 -week behavioral group therapy. The intervention was designed to improve $\mathrm{BoL}$ by helping participants to identify and effectively manage stressful experiences. It focused on treatment-related sequelae from PC. Differences in variables were compared between the beginning and end of the study by means of Student's t test for paired samples. Multiple analysis was carried out by stepwise multiple linear regression following bivariate Pearson's correlation analysis. This was achieved for all predictors (i.e. general health perception, ED and UI impact) and relevant covariates (i.e. age, work/retirement status, alcohol addiction, attitude towards cancer and surgery, sexual satisfaction, and future plans).

Results: In all patients the time elapsed from surgery to attending the behavioral group therapy exceeded 24 months. There was no significant correlation between this time and the predictor variables. General health perception scores decreased by the end of the study $(\mathrm{p}=0.000)$, as did the UI impact score $(\mathrm{p}=0.023)$, thus denoting improvement in both factors. The difference in UI impact scores correlated negatively and significantly with both age $(\mathrm{p}=0.04)$ and work/retirement $(\mathrm{p}=0.05)$. Multiple stepwise regression showed that age was the most important variable $\left(r^{2}=26.0 \%\right)$. Considering age and work/retirement simultaneously, there was an increase of $10.3 \%\left(r^{2}=36.3 \%\right)$. ED increased by the end of the study $(\mathrm{p}=0.000)$, and the difference between the ED scores correlated positively and significantly with sexual satisfaction alone $(\mathrm{p}=0.029)$, which signifies that previous sexual satisfaction had a positive influence over erectile dysfunction $\left(\mathrm{r}^{2}=15.8 \%\right)$.

Conclusion: 24-week behavioral group therapy was effective in improving the perceived $\mathrm{BoL}$ among men treated for PC. There were changes associated with the therapy, particularly the improvement in UI and ED.

Keywords: Behavioral group therapy. Prostate carcinoma. Quality of life. 
$\mathrm{L}$ os resultados sobre la calidad de vida relacionada a la salud (Health related quality of life-HRQoL) tienen un impacto sustancial en hombres que se enfrentan con la difícil decisión de someterse a tratamiento precoz de carcinoma prostático. Al final, la doble meta de curar el cáncer y mantener la calidad de vida es fundamental. Los hombres con cáncer de próstata tienden a priorizar acciones que puedan optimizar la calidad de vida más que a maximizar la supervivencia ${ }^{1}$.

El carcinoma prostático (CP) es el tumor sólido mas frecuente y es la segunda causa de muerte en hombres. La Prostatectomía Radical (PR) es el tratamiento primario para la enfermedad localizada. Aunque la PR es altamente efectiva para suprimir la recurrencia e incrementar la supervivencia ${ }^{2}$, los beneficios del tratamiento son casi siempre superados por la seria disminución en la calidad de vida primariamente asociada a los efectos secundarios del tratamiento, tales como incontinencia urinaria (IU) y disfunción eréctil (DE).

Se ha corroborado claramente la ventaja de realizar tratamientos psicológicos para mejorar la $\mathrm{GoL}$, reducir el stress y los efectos negativos en pacientes con cáncer. Estos tratamientos psicosociales están específicamente diseñados para cubrir las necesidades de pacientes con cáncer e incluyen terapias de grupo de apoyo, intervenciones educacionales y enfoques de intervención multimodal ${ }^{3,4}$. Los componentes efectivos de terapia incluyen: entrenamiento de relajación para baja estimulación, información sobre el tratamiento, un ambiente de apoyo emocional en donde los participantes puedan expresar sus temores y ansiedades; estrategias cognitivas y de comportamiento y apoyo social ${ }^{5}$.

Casi todos los programas de terapia enfatizan la restauración cognitiva, mejora la capacidad de respuesta, apoyo social, control de la ira y sentimientos negativos ${ }^{6}$.

Diversos estudios han demostrado que la participación en programas psicológicos proporcionan claros y duraderos beneficios para pacientes con cáncer, aliviando los síntomas relacionados con el tratamiento, reduciendo las consecuencias fisiológicas del stress y mejorando el carácter ${ }^{7}$. Estos resultados apoyan resultados previos en hombres tratados por CP precoz en el cual se estableció que los pacientes que participaban de programas multimodales tenían mejor salud mental, menos conflictos interpersona- les, mayor percepción sobre la salud y funcionalidad y menor estrés asociado a pensamientos negativos relacionados con los controles del cáncer ${ }^{8}$. En el presente estudio analizamos la eficacia de las terapias de grupo para mejorar la QoL de los hombres tratados por CP localizado. El diseño del estudio consistió en medidas repetidas con dos puntos en el tiempo (pre y post intervención). La hipótesis fue que los pacientes podrían percibir mejoría en la GoL después de realizar de terapia de grupo comparada con las mediciones realizadas antes de la intervención.

\section{MATERIALES Y MÉTODOS \\ Participantes}

Fueron reclutados para el estudio participantes entre aquellos que se atendieron en consulta ambulatoria en las unidades de urología y oncología del Hospital Universitario de la Unicamp. Para ser poder ser elegidos, los participantes deberían ser mayores de 50 años, haber sido sometido a una prostatectomía radical por lo menos en los últimos 24 meses y haber reportado efectos secundarios relacionados al tratamiento como incontinencia urinaria y disfunción eréctil de bajo nivel (pacientes usando solo 1 pañal al día y un IIEF-SF hasta 15) y que mostraran interés de participar dentro del grupo. Para participar bajo condiciones experimentales se requirió que los participantes estuvieran libres de deterioro cognitivo o síntomas psiquiátricos agudos incluyendo ataques de pánico, psicosis o adicción a alcohol o drogas y también que no padecieran de diabetes mellitus. Los pacientes fueron incluidos sólo si ellos no reportaban terapia adyuvante para $\mathrm{CP}$, IU o DE.

Los pacientes que reunieron los criterios de inclusión firmaron un consentimiento informado antes de la entrevista y completaron los cuestionarios de "The Kings Health Questionnaire (KHQ)" y el "International Index of Erectile Function (IIEF-SF)".

\section{Mediciones}

El impacto de la incontinencia urinaria (IU) sobre la calidad de vida fue evaluado de forma específica , con el "Kings Health Questionnaire (KHQ)", el cual es una herramienta ampliamente aplicada compuesta de 21 preguntas con de diferentes categorías: percepción de la salud general, impacto de la incontinencia urinaria, limitaciones físicas, relaciones personales, emociones, sueño y vitalidad. 
También contiene dos escalas de evaluación de IU: "medidas de severidad", las cuales evalúan la seriedad de la IU y la "escala de síntomas urinarios" que evalúa la presencia e intensidad de la IU. El cuestionario tiene una puntuación para cada escala de manera separada. No se realizó medida objetiva de la incontinencia, debido a que el principal propósito a evaluar era sólo la percepción de los pacientes sobre su calidad de vida en relación a IU.

La disfunción eréctil fue investigada usando el "International Index of Erectile Function (IIEF-SF)", diseñado para evaluar dificultades de erección. Se validaron ambos cuestionarios.

\section{Procedimientos}

Los pacientes asistieron a sesiones semanales de terapia de grupo (10 pacientes en cada grupo) con una duración de 90 minutos. El tiempo de tratamiento fue de 6 meses. Tres meses después de finalizar la terapia los pacientes fueron reevaluados a través de los mismos procedimientos utilizados.

\section{Condición Experimental}

En el presente estudio, el grupo de terapia de 24 semanas se reunión una vez a la semana durante 90 minutos, incluyendo una parte de aprendizaje. A los participantes se les enseño una variedad de herramientas para el manejo del stress incluyendo la identificación de pensamientos distorsionados, con reemplazo de pensamientos racionales, manejo de la ira, reafirmación personal, utilización del apoyo social... Los componentes de la enseñanza fueron dispuestos de tal forma que cada sesión se relacionaba con el material presentado la semana anterior. Los tópicos incluyeron enfoques cognitivos de comportamiento para el manejo del stress y también más material informativo relacionado al diagnostico y tratamiento (es decir la fisiología del CP y la mecánica y el tratamiento de la disfunción eréctil). Semanalmente se motivó a los participantes a practicar actividad física diaria.

\section{Análisis Estadístico}

Todos los análisis estadísticos fueron realizados con el software SPSS (versión 12.0; SPSS Inc., Chicago, IL). Las diferentes variables fueron comparadas al inicio y al final del estudio por medio del test de Student para muestras pareadas. El Análisis múltiple se llevo a cabo usando regresión linear múltiple siguiendo el análisis de correlación bivariada de Pearson. Las variables que presentaban diferencias significativas fueron tomadas como variables dependientes y fueron evaluadas juntamente con la edad, hipertensión, trabajo/retiro, adicción al alcohol, actitudes hacia el cáncer, actitudes hacia la cirugía, satisfacción sexual y planes futuros... En todos los casos la significación estadística se obtuvo cuando $\mathrm{p} \leq 0,05$.

\section{RESULTADOS}

Los participantes en el estudio fueron 30 hombres a quienes se les realizo prostatectomía radical para $\mathrm{CP}$ localizado en los últimos 24 meses. De ellos fueron caucásicos $(66,7 \%)$, seguidos por negros $(30,0 \%)$. La edad media fue de 62,5 años ( $\mathrm{SD}=7,2$ años). Los ingresos anuales del $86,7 \%$ de los participantes fueron menores de \$100.00, y el 83,3\% de ellos sólo tenían 4 años de estudios primarios. Al final del estudio $(\mathrm{p}=0,000)$, mostraron mejoría en ambos factores (IU y DE). La diferencia en el score de impacto de IU correlacionó negativamente y significativamente con la edad $(p=0,04)$ y trabajo/retiro $(p=0,05)$. El análisis de regresión múltiple mostró que la edad fue la variable más importante $\left(r^{2}=26,0 \%\right)$. Considerando la edad y trabajo/retiro simultáneamente hubo un incremento de $10,3 \%\left(r^{2}=36,3 \%\right)$.

La disfunción eréctil (DE) se incrementó al final del estudio ( $\mathrm{p}=0,000)$, y la diferencia entre los scores de DE correlacionaron positivamente y significativamente sólo con satisfacción sexual ( $\mathrm{p}=0,029$ ), lo cual significa que la satisfacción sexual previa tenia una influencia positiva sobre la disfunción eréctil $\left(\mathrm{r}^{2}=15,8 \%\right)$.

La Tabla 1 muestra los valores medios para las variables predictivas previas y los valores de $\mathrm{p}$ para la diferencia antes y después del tratamiento.

Tabla 1. Comparación del valor significativo por las variables predictivas entre el comienzo y el final de comportamiento del grupo bajo terapia.

\begin{tabular}{|c|c|c|c|c|c|}
\hline \multirow[t]{2}{*}{ Variable } & \multicolumn{2}{|c|}{$\begin{array}{c}\text { Antes del } \\
\text { tratamiento }\end{array}$} & \multicolumn{2}{|c|}{$\begin{array}{l}\text { Después del } \\
\text { tratamiento }\end{array}$} & \multirow[t]{2}{*}{$\begin{array}{l}\text { Valor } \\
\text { de p }\end{array}$} \\
\hline & Media & sd & Media & sd & \\
\hline DE* & 9,7 & 0,9 & 13,2 & 1,1 & 0,000 \\
\hline $\begin{array}{l}\text { Percepción de } \\
\text { la salud general }\end{array}$ & 43,3 & 2,9 & 30,0 & 3,0 & 0,000 \\
\hline Impacto IU** & 37,8 & 6,1 & 26,7 & 4,9 & 0,023 \\
\hline
\end{tabular}

*DE: disfunción eréctil.

**IU: Incontinencia urinaria. 


\section{DISCUSIÓN}

Este estudio analiza cómo una terapia de grupo de 24 semanas mejora la calidad de vida entre hombres tratados por CP. Trabajos previos han reportado la eficacia de intervenciones psicosociales reduciendo el estrés y los efectos negativos así como mejorando la calidad de vida entre varias poblaciones de pacientes con cáncer ${ }^{7,9,10}$.

Casi todos los estudios se han conducido primariamente a mujeres después del tratamiento para cáncer de mama, mostrando en recientes Investigaciones la efectividad de tratamientos psicoeducacionales que mejoran la tolerancia y en una menor proporción el funcionamiento sexual ${ }^{11 .}$

En hombres con CP. Lepore ${ }^{8}$ reportó que los que habian participado en una terapia de grupo de 6 semanas mejoraban en algunos índices incluyendo salud mental y control percibido y redujeron sus conflictos interpersonales y nerviosismo asociados a pensamientos negativos relacionados con el cáncer. Aunque estos estudios empiezan a sugerir el beneficio de los tratamientos psicosociales aun es limitada la presencia de estudios randomizados. Además, los mecanismos por el cual las intervenciones psicosociales pueden mejorar la GoL de hombres con CP están por identificarse.

Trabajos previos han sugerido que las secuelas del tratamiento de CP parecen tener poco impacto sobre la GoL general ${ }^{12}$, a pesar del hecho que los paciente han informado de nerviosismo y molestias por deterioros asociados al tratamiento ${ }^{13,14}$. Esa disparidad de argumentos han sido evaluados en estudios que han sugerido que los estos pacientes minimizan el impacto del CP en su vida diaria ${ }^{15}$. Un gran porcentaje de pacientes con una pobre funcionalidad caracterizada por disminución de la función eréctil y urinaria reportan que esto no es problema para ellos o sólo un pequeño problema. Así aunque padezcan de escapes de orina diaria o frecuente o no tengan erecciones, parecen tener un mejor ajuste a estos situaciones. Además, estos pacientes desean aparecer ante otros pacientes y ante ellos mismos como que enfrentan el problema de manera normal. Es sabido que los hombres tienden a soslayar problemas para evitar dañar a otros o parecer como vulnerables, reduciendo de esa manera efectos negativos e intentando mantener una auto imagen positiva ${ }^{16}$. A pesar del hecho que estas estrategias pueden ser positivas y pueden llevar a una adecua- da GoL, también puede ser cierto el hecho que tales estrategias cognitivas, afectivas y del comportamiento tengan, aunque ocultas, consecuencias significativas (efectos negativos no expresados como ira, aislamiento social, etc.). Consecuentemente, hemos evaluado la eficacia de la intervención diseñada para mejorar la $\mathrm{GoL}$ proporcionando distintas estrategias psicosociales.

En el actual estudio encontramos que la participación en nuestras terapias de grupo mejora significativamente la QoL general. La falta de correlación entre el tiempo trascurrido de la cirugía al inicio del tratamiento y las variables predictivas hacen que pueda concluirse que la mejora aquí descrita es mayormente debida al efecto de la intervención psicosocial más que a la evolución natural en el tiempo.

Aunque esta hipótesis puede ser apoyada estadísticamente, este estudio se basó en una pequeña muestra en un periodo de 9 meses (desde la preintervención a la post-intervención), lo cual limita la capacidad de generalizar nuestros hallazgos. Futuros estudios deben permitir repetir estos hallazgos en muestras mayores, en periodos más largos de tiempo y con una muestra adecuada. Es esencial que los futuros estudios incidan en el efecto a largo plazo de las intervenciones psicosociales en la GoL y los procesos de la enfermedad en orden a establecer la significación clínica de los beneficios de tales intervenciones en un creciente número de hombres tratados por CP.

\section{REFERENCIAS}

1. Litwin MS. Measuring health related quality of life in men with prostate cancer. J Urol.1994;152(5 Pt 2):1882-1887.

2. Neulander EZ, Soloway MS. Failure after radical prostatectomy. Urology. 2003; 61(1):30-36.

3. Spiegel D, Yalom ID. A support group for dying patients. Int J Group Psychother. 1978;28(2):233-245.

4. Fawzy FI, Fawzy NW, Huym CS, Weeler JF. Brief coping oriented therapy for patients with malignant melanoma. In: Spira JL, editor. Group therapy for medically ill patients. New York: Guilford Press. 1997; 133-164.

5. Meyer TJ, Mark, MM. Effects of psychosocial interventions with adult cancer patients: a meta-analysis of randomized experiments. Health Psychol. 1995;14(2):101-108.

6. Rehse B, Pukrop R. Effects of psychosocial interventions on quality of life in adult cancer patients: meta analysis of 37 published control outcomes studies. Patient Educ Counsel. 2002; 1658:1-8.

7. Mishel MH, Belyea M, Germino BB, Stewart JL, Bailey DE Jr, Robertson C, et al: Helping patients with localized prostate carcinoma manage uncertainty and treatment side effects. Cancer. 2002; 94(6): 1854-1866. 
8. Lepore SJ. A social-cognitive processing model of emotional adjustment to cancer. In: Baum A, Andersen BL, editors. Psychosocial interventions in cancer. Washington, DC: American psychological Association. 2001;99-116.

9. Yalom ID. The theory and practice of group psychotherapy. Basic Books, 1983.

10. Sheard T, Maguire P. The effect of psychological interventions on anxiety and depression in cancer patients: results of two meta-analyses. Br J Cancer. 1999;80(11):1770-1780.

11. Penedo FJ, Dahn JR, Molton I, Gonzalez JS, Kinsinger D, Roos BA, et al. Cognitive-behavioral stress management improves stress management skills and quality of life in men recovering from treatment of prostate carcinoma. Cancer. 2004;100(1): 192-200.

12. Eton DT, Lepore SJ. Prostate cancer and health-related quality of life: a review of the literature. Psychooncology. 2002;11(4): 307-326.

13. Schover LR, Fouladi RT, Warneke CL, Neese L, Klein EA, Zippe C, et al. Defining sexual outcomes after treatment for localized prostate carcinoma. Cancer. 2002:95(8):1773-1785.
14. Wei JT, Dunn RL, Sandler HM, McLaughlin W, Montie JE, Litwin MS, et al. Comprehensive comparison of health-related quality of life after contemporary therapies for localized prostate cancer. J Clin Oncol. 2002;20(2):557-566.

15. Hoffman RM, Hunt WC, Gilliland FD, Stephenson RA, Potosky AL. Patient satisfaction with treatment decisions for clinically localized prostate carcinoma: results from the prostate cancer outcomes study. Cancer. 2003;97(7):1653-1662.

16. Kosberg JI. The invisibility of older men in gerontology. Gerontol Geriatr Educ. 2002;22:27.

Correspondencia autor: Dr. Lygia F.G. Perchon

Division of Geriatric Urology

Federal University of São Paulo. Brazil

E-mail autor: lygiaperchon@terra.com.br

Información artículo: Original -

Trabajo recibido: septiembre 2007

Trabajo aceptado: noviembre 2007 